\title{
ENSEMBLE POUR LES MESURES EN TEMPS-DE-VOL DES NEUTRONS RAPIDES
}

\author{
C. LE RIGOLEUR et D. ABRAMSON, \\ C.E.N., Gadarache.
}

\begin{abstract}
Résumé. - Nous décrivons un ensemble expérimental disposé auprès du faisceau pulsé regroupé du Van de Graaff de $5 \mathrm{MeV}$ de Cadarache, piloté par un calculateur CII 90-10 et destiné aux mesures neutroniques par temps-de-vol. Il comprend cinq détecteurs de neutrons rapides et différents moniteurs. De plus, un électroaimant permettant la précession de $\pi$ du spin des neutrons d'énergie jusqu'à $4 \mathrm{MeV}$ a été mis au point pour des mesures de polarisation.

Abstract. - We describe an experimental set-up, located near the $5 \mathrm{MeV}$ pulsed and bunched Van de Graaff at Cadarache operated by a CII 90-10 computer and intended for neutron time-of-flight measurements. It includes five fast neutron detectors and various monitors. In addition a precession electromagnet was designed for neutron polarisation measurements.

衰
\end{abstract}

I. Ensemble à cinq détecteurs de neutrons rapides. - I.1. Détecteurs et collimation ( fig. 1). - Ces détecteurs sont des scintillateurs liquides à protons de

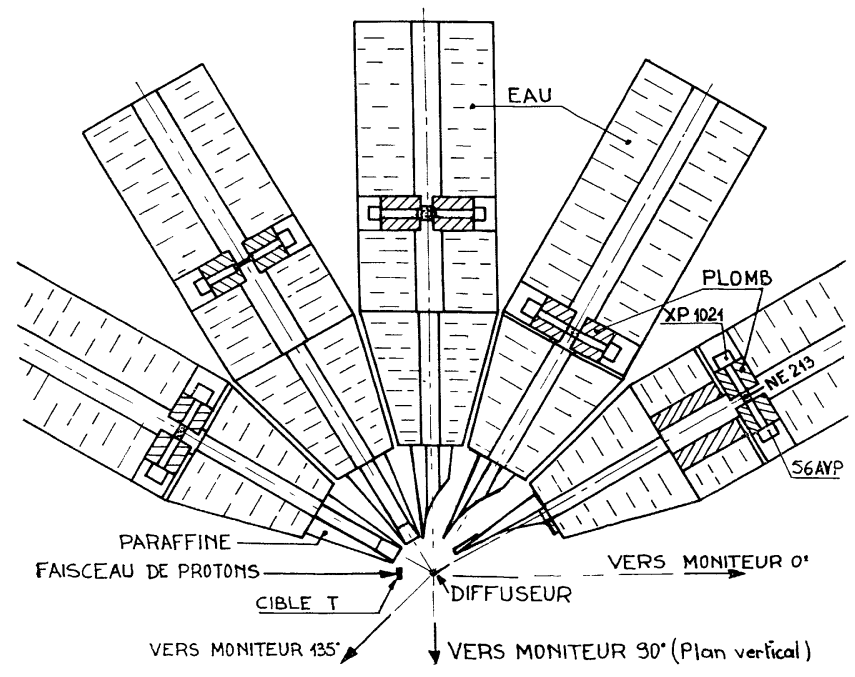

FIG. 1.

recul NE 213. Chaque scintillateur est logé dans une protection en plomb, elle-même disposée dans une cuve de fer pleine d'eau, devant laquelle se trouve un cône en paraffine (ou une portion de cône). Cette collimation est voisine de celle réalisée par A. B. Smith à Argonne. En améliorant sous le faisceau de neutrons la forme de ces protections de paraffine pour les détecteurs situés le plus en avant, nous avons obtenu des bruits de fond très petits. Les détecteurs sont à $30^{\circ}$ les uns des autres. Comme l'ensemble des détecteurs et de leurs protections est disposé sur un chariot tournant, on peut mesurer des distributions angulaires de $25^{\circ}$ à $155^{\circ}$. La position du chariot peut être télécommandée par le calculateur. La distance diffuseur-détecteur peut varier de 1,50 à $4 \mathrm{~m}$.

I.2. ÉleEGtronique ( fig. 2). - Les neutrons sont sélectionnés par les méthodes de temps-de-vol et de discrimination $\gamma$-n. Chaque détecteur est vu par deux pho-

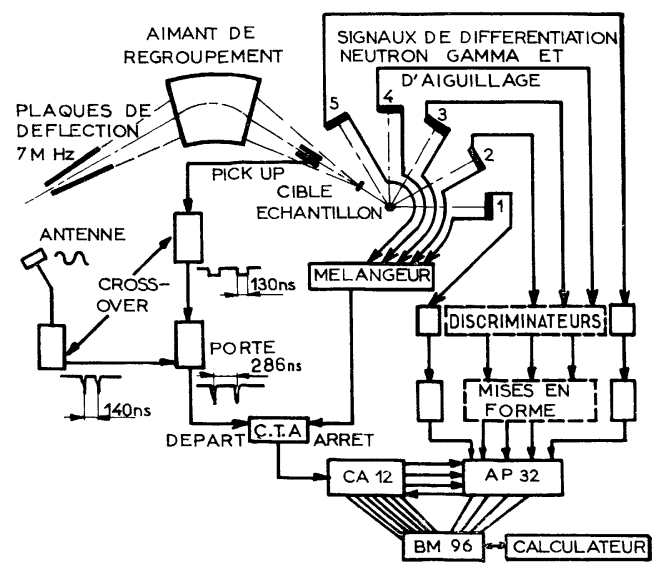

Fig. 2. - Ensemble de 5 détecteurs de neutrons dispositif électronique.

tomultiplicateurs. Le premier, un XP 1021, est un photomultiplicateur rapide qui sert à déterminer le temps de détection du neutron. Le second, un 56 AVP 03, permet la discrimination $\gamma$-n par la méthode d'Owen. Le seuil de détection des neutrons en énergie est d'environ $150 \mathrm{keV}$. Les impulsions provenant de chacun des XP 1021 sont mélangées grâce à un système à 
diodes rapides et envoyées sur la voie « arrêt » d'un convertisseur temps-amplitude dont la voie « départ » est déclenchée par une impulsion périodique provenant du faisceau pulsé et regroupé du Van de Graaff. Chacune des impulsions proverıant des 56 AVP 03 permet la différenciation $\gamma$-n après avoir été discriminée en amplitude et est envoyée dans l'une des voies du châssis d'aiguillage AP 32 (Intertechnique). L'impulsion fournie par le CTA est envoyée dans un convertisseur d'amplitude CA 12 (Intertechnique). Si cette impulsion est en coïncidence avec l'une des impulsions attaquant l'AP 32, elle est analysée en amplitude. Le résultat de la conversion est aiguillé par l'AP 32 dans un sous-groupe de canaux d'un bloc-mémoire BM 96.

La résolution en temps est de l'ordre de 2,5 ns avec un scintillateur cylindrique de $1^{\prime \prime} 1 / 2$ de diamètre et $2^{\prime \prime}$ de longueur, et des neutrons de $2,5 \mathrm{MeV}$.

Les détecteurs sont stabilisés en température par une circulation d'eau à $22^{\circ}$ autour des photomultiplicateurs. La variation de l'efficacité de détection des neutrons d'une source Po-Be est inférieure à $\pm 1,5 \%$ sur trois mois.

I.3. Moniteurs. - L'installation comprend différents moniteurs. L'un d'entre eux, un détecteur directionnel à $\mathrm{BF}_{3}$ dont l'efficacité est connue, fait l'objet d'une autre communication à ce congrès. Il est placé à $0^{\circ}$ dans l'axe du faisceau. Un autre, semblable, est placé à $90^{\circ}$. Enfin, un troisième placé à $135^{\circ}$, constitué par un scintillateur plastique, est utilisé en tempsde-vol.

I.4. Mesures. - Les mesures peuvent être conduites entièrement par le calculateur à qui l'on indique préalablement les conditions de l'expérience. Les résultats sont lus par le calculateur, transférés sur bande magnétique et analysés ultérieurement.

Un tel ensemble joint au calculateur permet ainsi une utilisation rationnelle du faisceau. Nous avons effectué des mesures de distribution angulaire de neutrons diffusés élastiquement par le lithium-6 de 2 à $2,9 \mathrm{MeV}$.

II . Electroaimant de précession de spin. - Enfin, dans le but d'effectuer des mesures de polarisation, nous avons réalisé un électroaimant qui permet de faire pivoter de $180^{\circ}$ la polarisation d'un faisceau de neutrons d'énergie maximale égale à $4,3 \mathrm{MeV}$. Les dépolarisations introduites par les inhomogénéités de champ et de rémanence sont négligeables (inférieures à $0,5 \%$ ). 